\title{
Four-factor prothrombin complex concentrate improves thrombin generation and prothrombin time in patients with bleeding complications related to rivaroxaban: a single-center pilot trial
}

\author{
Bettina Schenk ${ }^{1 *}$ (D) Stephanie Goerke ${ }^{2,3}$, Ronny Beer ${ }^{4}$, Raimund Helbok ${ }^{4}$, Dietmar Fries ${ }^{1}$ and Mirjam Bachler ${ }^{1,5}$
}

\begin{abstract}
Background: Direct oral anticoagulants (DOACs) pose a great challenge for physicians in life-threatening bleeding events. The aim of this study was to test the efficacy of reversing the DOAC rivaroxaban using four-factor PCC (prothrombin complex concentrate), a non-specific reversing agent.

Methods: Patients with life-threatening bleeding events during rivaroxaban treatment were included and administered $25 \mathrm{U} \mathrm{kg}^{-1}$ of PCC. Blood samples were collected immediately prior to as well as after PCC treatment at predefined time intervals. The primary endpoint was defined as the difference in thrombin generation (TG) parameters ETP (endogenous thrombin potential) and $C_{\max }$ (peak thrombin generation) prior to and ten minutes subsequent to PCC treatment.

Results: Thirteen patients, of whom the majority suffered from intra-cranial haemorrhage (ICH) or subdural haemorrhage (SDH), were included and administered PCC. The results show that the ETP (TG) significantly ( $p=0.001)$ improved by $68 \%$ and $C_{\max }(\mathrm{TG})$ by $54 \%(p=0.001)$ during PCC treatment. In addition, the Quick value (prothrombin time: Quick ${ }^{P T}$ ) significantly improved by $28 \%$ and the activated partial thromboplastin time (aPTT) was decreased by $7 \%$ ten minutes after PCC administration. $C_{\max }$ was reduced at baseline, but not ETP, aPTT or Quick ${ }^{\mathrm{PT}}$. Lag time until initiation $\left(T G, t_{\text {lag }}\right)$, thromboelastometry clotting time $\left(\mathrm{CT}_{\text {EXTEM }}\right)$ and time to peak $\left(T G, t_{\text {max }}\right)$ correlated best with measured rivaroxaban levels and were out of normal ranges at baseline, but did not improve after PCC administration. In 77\% of the patients bleeding (ICH/SDH-progression) ceased following PCC administration. During the study three participants passed away due to other complications not related to PCC treatment. The possibility of thrombosis formation was also evaluated seven days after administering PCC and no thromboses were found.

Conclusions: This study shows that use of PCC improved ETP, $C_{\max }$, Quick ${ }^{\mathrm{PT}}$ and aPTT. However, of these parameters, only $C_{\max }$ was reduced at the defined baseline. It can be concluded that $C T_{\text {EXTEM, }}, t_{\text {lag }}$ and $t_{\max }$ correlated best with the measured rivaroxaban levels. The study drug was found to be safe. Nonetheless, additional studies specifically targeting assessment of clinical endpoints should be performed to further confirm these findings.
\end{abstract}

Clinical trial registration: EudraCT trial No. 2013-004484-31.

\footnotetext{
* Correspondence: bettina.schenk@i-med.ac.at

1 Department of General and Surgical Intensive Care Medicine, Medical

University of Innsbruck, Anichstr. 35, 6020 Innsbruck, Austria

Full list of author information is available at the end of the article
} 


\section{Background}

Rivaroxaban (Xarelto ${ }^{\circ}$, Bayer, Germany) is used as thrombosis prophylaxis and therapy instead of anticoagulants such as unfractionated heparin, low molecular weight heparin or vitamin $\mathrm{K}$ antagonists, because it is considered to have a wider therapeutic range and a more predictable dose-response [1-3]. Although bleeding and bleeding complications in trauma and surgery are the most common complications/side-effects [4], no evidence-based reversal strategy is available for a bleeding patient taking rivaroxaban $[5,6]$. A specific antidote called Andexanet alfa is in development, but not yet clinically available [7]. Therefore, the off-label use of coagulation factor concentrates, particularly four-factor prothrombin complex concentrate, in the management of rivaroxaban-related, life-threatening bleeding is the most recommended/used therapy $[8,9]$ : In healthy male volunteers, PCC was seen to reverse the anticoagulant effect of rivaroxaban $[10,11]$. Especially for the treatment of intracranial haemorrhage (ICH), PCC seems to be a promising potential reversing agent [12-14]. However, managing life-threatening bleeding events under DOAC remains a challenge for physicians since in vivo data regarding efficacy of anticoagulation reversal are still lacking.

Therefore, the aim of this study was to evaluate the effect of PCC on coagulation status after life-threatening bleeding events in patients treated with rivaroxaban.

\section{Methods}

\section{Ethics committee approval}

This study was approved by the Human Subjects Review Board of the Medical University of Innsbruck, Austria (Ref.: UN2013-0048), as well as by the national competent authority (Bundesamt für Sicherheit im Gesundheitswesen, BASG, Vienna, Austria, Ref.: LCM-718199) and registered with EudraCT (Ref.: 2013-004484-31). Written informed consent was obtained from all study participants. Patients, who for medical reasons (unconscious, shock, sedated, etc.) were not able to give their consent to participate in this clinical trial, were enrolled based on the principle of deferred consent according to $\$$ 43a (1), Austrian Medicinal Products Law ("Österreichisches Arzneimittelgesetz").

The study was performed in compliance with the Declaration of Helsinki guidelines regarding ethical principles for medical research involving human subjects and followed Good Clinical Practice as defined by the International Conference on Harmonization (ICH-GCP).

\section{Study design and setting}

The present study is a single-centre, analytic, observational, prospective, open-labelled, single-armed, non-commercial clinical pilot trial.

\section{Selection of participants}

Inclusion criteria were age over 18 years, patients receiving rivaroxaban and with life-threatening bleeding and the need for reversal and/or patients needing acute reversal of the anticoagulation effects of rivaroxaban. Patients who had already been administered pro-coagulant therapies including recombinant activated factor seven, activated prothrombin complex concentrates or other coagulation factor concentrates (except fibrinogen concentrate, DDAVP and tranexamic acid) were not included. Further exclusion criteria were: a greater risk for thromboembolic events than for bleeding, pregnancy, suspected or confirmed sepsis, recent history of thromboembolic events, and active participation in another clinical trial or refusal to participate.

\section{Interventions}

The investigational medicinal product (four-factor PCC) was generously provided by CSL Behring, Marburg, Germany (Beriplex $\left.{ }^{\ominus} \mathrm{P} / \mathrm{N} 500 \mathrm{IU}\right)$. PCC was administered i.v. at a dosage of $25 \mathrm{IU} / \mathrm{kg} \mathrm{BW}$ as bolus and/or continuous infusion. If bleeding continued after the first dosage, a second dosage of $25 \mathrm{IU} / \mathrm{kg} \mathrm{BW}$ had to be administered. However, for all of the included patients only the first dosage of PCC was necessary. The maximum total allowed dose was 5000 IU.

Study assessments were performed as follows: Visit 1 (V1, baseline) was performed immediately before administration of four-factor PCC (25 IU/kg BW). Ten minutes after the end of PCC administration Visit 2 was conducted (V2). Thereafter, Visits were calculated as V3: +1 h, V4: +3 h, V5: +6 h, V6: +12 h, V7: +24 h, V8: +2 days, V9: +4 days, V10: +7 days, V11 +30 days. At Visit 11 (30 days after V2) the patient was interviewed to evaluate his health status. Between days 5 and 7 an ultrasound examination of the leg arteries and veins was performed to screen for thrombosis. At every visit bleeding and thrombosis status were assessed, laboratory measurements performed and vital signs documented.

Blood coagulation assays were performed and blood coagulation factor concentrations determined using vacutainer tubes containing $1.106 \mathrm{~mol} \mathrm{l}^{-1}$ trisodium citrate solution; blood samples for blood cell count were taken using vacutainer tubes containing $1.6 \mathrm{mg} \mathrm{ml}^{-1} 3 \mathrm{~K}$ EDTA. All blood sampling tubes were purchased from Sarstedt, Nümbrecht, Germany.

All coagulation analyses were performed immediately after blood draw, except thrombin generation. Plasma for thrombin generation assays was immediately frozen at $-80{ }^{\circ} \mathrm{C}$ (or at $-20{ }^{\circ} \mathrm{C}$ for a maximum of seven days) and thawed immediately before being analysed.

\section{Methods and measurements}

ROTEM $^{\curvearrowleft}$ parameters were determined using a ROTEM $^{\odot}$ gamma analyser (TEM Innovations $\mathrm{GmbH}$, Munich, 
Germany). All tests were performed according to manufacturer's instructions in whole blood immediately after blood sampling and using the specific liquid reagents provided by the manufacturer for EXTEM (extrinsically activated assay with tissue factor) and INTEM (intrinsically activated test using kaolin) measurements.

Thrombin generation measurements were performed using the Innovance ETP assay (Siemens, Marburg, Germany) on an automated coagulation analyser (BCS XP, Siemens, Marburg, Germany). Coagulation was activated by adding phospholipids, human recombinant tissue factor (in a concentration of $600-850 \mathrm{pmol} \mathrm{l}^{-1}$ ) and calcium ions to platelet-poor plasma. The generated thrombin cleaves a chromogenic substrate (H-b-Ala-Gly-Arg-pNA) and the turnover of the substrate is recorded over time. The final substrate concentration is $733 \mathrm{nmol} \mathrm{l} \mathrm{l}^{-1}$ and $19 \mathrm{mmol} \mathrm{l}^{-1}$ $\mathrm{CaCl}$. The original curve is corrected for the estimated $\alpha$ macroglobulin-bound thrombin activity. From this curve the following parameters can be obtained: total amount of generated thrombin present in the reaction from the point of initiation until return to baseline, which is measured by the calculated increase in the extinction rate (measured in milliextinctions or $\mathrm{mE}$ ), and also known as "endogenous thrombin potential" (ETP), sometimes referred to as "area under the curve" (AUC), peak thrombin generation $\left(C_{\max }\right)$ (the maximum of the first derivation of the ETP), lag phase until initiation $\left(t_{\mathrm{lag}}\right)$, and time to peak thrombin activity $\left(t_{\max }\right)$.

PT, aPTT, d-dimers, vWF, plasminogen, single factor levels (FII, FV, FVII, FVIII, FIX, FX, FXI, FXII and FXIII) and antithrombin activity were determined with proper reagents (PT: Thromborel ${ }^{\circ}$ S; aPTT: Pathromtin ${ }^{\circ} \mathrm{SL}$; DDimers: INNOVANCE ${ }^{\oplus}$ D-Dimer; vWF: vWF $\mathrm{Ag}^{\oplus}$, Innovance ${ }^{\circ}$ VWF Ac, Plasminogen: Berichrom ${ }^{\circ}$ Plasminogen; factor II, V, VII, X: Factor-deficient plasma via Thromborel $^{\bullet}$ S; factor VIII, IX, XI, XII: Factor-deficient plasma via Pathromtin ${ }^{\circ}$ SL; antithrombin III Berichrom ${ }^{\circ}$ Antithrombin III) on an automated coagulation analyser (reagents and BCSxp, Siemens, Marburg, Germany). The pondus Hydrogenii $(\mathrm{pH})$ value was measured with proper reagents on an automated blood gas analyser (ABL800, Drott Medizintechnik GmbH, Austria). CRP was measured using an immunoturbidimetric assay (Tina-quant ${ }^{\circ}$ CRPLX, Roche Diagnostics). Rivaroxaban levels were also measured on the BCSxp using a chromogenic assay calibrated for rivaroxaban (BIOPHEN ${ }^{\circ}$ DiXa-I, CoaChrom Diagnostica, Neuville-sur-Oise, France). Reference ranges were taken from the respective product specification files of the test reagents used (compare above, all reagents and machines with CE certification) or from the respective standard references [15].

\section{Outcomes}

Primary objective was assessment of the coagulation status after administration of PCC. Coagulation measurements included PT, aPTT, endogenous thrombin potential (ETP), thromboelastometry $\left(\right.$ ROTEM $\left.^{\circ}\right)$, single factor concentrations, blood cell count and the rivaroxaban level.

Based on previous investigations [16-20], the primary endpoint was defined as the difference in thrombin generation parameters ETP and $\mathrm{C}_{\max }$ between V1 and V2.

Secondary endpoints were the development of the following parameters from V1 to V10 and their correlation with measured rivaroxaban levels: thrombin generation, single factor profiles, standard coagulation tests PT, aPTT, fibrinogen, AT, and thromboelastometry (ROTEM $\left.{ }^{\circ}\right)$.

\section{Analysis}

A descriptive analysis of all measured blood characteristics was performed. The Wilcoxon signed-rank test was used to evaluate the primary endpoint and all other differences in coagulation measurements between baseline and samples from the same patient at V2 to V10. To evaluate correlations between rivaroxaban level and blood coagulation assays and coagulation factor activities Pearson's correlation was used. $P$ values $\leq 0.05$ were considered statistically significant. Statistical analyses were performed using STATISTICA 10 software (StatSoft Europe GmbH, Hamburg, Germany).

\section{Results}

\section{Characteristics of study subjects}

Fourteen patients ( 5 female, 9 male) aged between 47 and 96 years (median 80 years) were enrolled at the Medical University of Innsbruck. One patient (male) had to be excluded from analysis because he withdrew consent. Median weight was $76 \mathrm{~kg}$ (range $52 \mathrm{~kg}$ to $99 \mathrm{~kg}$ ) and median height $170 \mathrm{~cm}$ (range $156 \mathrm{~cm}$ to $183 \mathrm{~cm}$ ). Average body mass index was 25 (range 18 to 34 ).

The first visit to the first patient (FPFV) was in August 2014, last patient last visit (LPLV) was in October 2016. Underlying disease was mostly intracerebral haemorrhage (ICH, six patients), four patients suffered from subdural hematoma (SDH), one patient suffered from haemorrhage after removing the urinary catheter, one during laparotomy, and one suffered from gastrointestinal bleeding.

Prior medication (within seven days before V1), besides rivaroxaban (100\%), was recorded if patients received additional antiplatelet medication (2 patients), procoagulatory medication (no patient) or anticoagulatory medication (no patient).

Median amount of administered PCC was 2000 IU (range 1500 to $2400 \mathrm{IU}$ ).

Concomitant medication (from V1 to V7), with potential influence on coagulation, included anticoagulant medication (12 patients), catecholamines (10 patients), antibiotics (6 patients), procoagulant medication (1 patient), anti-fibrinolytics (1 patient) and blood products (1 
patient): ten patients received noradrenaline, seven patients enoxaparin, two patients rivaroxaban, and one patient each received danaparoid, fibrinogen concentrate, tranexamic acid and erythrocyte concentrate.

\section{Safety}

Three subjects died during study participation, one from septic shock (multi-organ failure), one due to progressive cancer and one from ICH. All deaths were considered to be unrelated to the study medication. Of the thirteen study patients three showed signs of re-bleeding (progressive $\mathrm{ICH}$ ) after administration of PCC. Other serious adverse events (SAEs) were an epileptic event, ischemia caused by thromboembolic closure of the femoral artery bifurcation (left), sepsis and embolic arteria cerebri anterior infarct (one patient). Both thromboembolic SAEs occurred only after day 7 (thrombotic screening via duplex ultrasound).

\section{Main results}

Baseline For baseline values of laboratory parameters other than coagulation measurements, please refer to Table 1.

Mean rivaroxaban level at V1 was $120 \mathrm{ng} \mathrm{ml}^{-1}$ (95\% confidential interval 48 to $192 \mathrm{ng} \mathrm{ml}^{-1}$ ). The course of measured rivaroxaban levels can be seen in Fig. 1. Standard laboratory parameters aPTT and PT (Quick value) were in normal range. Thromboelastometry EXTEM clotting time $\left(\mathrm{CT}_{\text {EXTEM }}\right)$ was prolonged, $\mathrm{C}_{\max }(\mathrm{TG})$ was reduced and ETP (TG) was in normal range. Moreover, C-reactive protein (CRP) levels were increased, as were d-dimers, FVIII levels and vWF levels, whereas erythrocyte and leukocyte counts were below reference ranges for healthy subjects. Blood loss was 800 and $750 \mathrm{~cm}^{3}$ for patients who were bleeding externally, whereas in the case of ICH intracerebral blood volume was $0.02 \mathrm{~cm}^{3}$, $8 \mathrm{~cm}^{3}, 12 \mathrm{~cm}^{3}, 34 \mathrm{~cm}^{3}, 43 \mathrm{~cm}^{3}$ and $99 \mathrm{~cm}^{3}$, respectively. Regarding SDH, blood loss volume was $3 \mathrm{~cm}^{3}, 11 \mathrm{~cm}^{3}$, $33 \mathrm{~cm}^{3}$ and $116 \mathrm{~cm}^{3}$, respectively. In the case of gastrointestinal bleeding blood loss was not measured. All baseline coagulation laboratory measurements can be seen from Table 2 .

\section{Primary endpoint}

All included patients reached the primary endpoint (difference in thrombin generation between V1 and V2). The ETP significantly $(p=0.001)$ increased by $68 \%$ as a result of administration of PCC (median amount of administered PCC: 2000 IU [range 1500-2400 IU]) from $316 \mathrm{mE}$ at $\mathrm{V} 1$ to $530 \mathrm{mE}$ at $\mathrm{V} 2 . \mathrm{C}_{\max }$ significantly $(p=$ 0.001 ) improved by $54 \%$ from $80 \mathrm{mE} \mathrm{min}^{-1}$ (V1) to $123 \mathrm{mE} \mathrm{min}^{-1}$ (V2). $t_{\text {lag }}$ and $t_{\max }$ did not show a significant difference between V1 and V2 (Fig. 2a and b).
Table 1 Baseline laboratory parameters of all included patients

\begin{tabular}{|c|c|c|c|c|c|}
\hline Parameter & Unit & Median & Min & Max & Reference range \\
\hline Rivaroxaban & $\mathrm{ng} \mathrm{ml}^{-1}$ & 103 & 20 & 425 & \\
\hline $\mathrm{CRP}^{\mathrm{a}}$ & $\mathrm{mg} \mathrm{dl}^{-1}$ & 0.7 & 0.1 & 16.5 & $0.0-0.5$ \\
\hline $\mathrm{pH}$ & & 7.42 & 7.33 & 7.49 & $7.37-7.45$ \\
\hline FI (Clauss) & $\mathrm{mg} \mathrm{dl}^{-1}$ & 303 & 240 & 524 & $210-400$ \\
\hline FI (immun) & $m g d^{-1}$ & 365 & 272 & 591 & $200-400$ \\
\hline ATIII (FIla) & $\%$ & 86 & 47 & 101 & 79-112 \\
\hline D-Dim ${ }^{a}$ & $\left.\mu g\right|^{-1}$ & 994 & 191 & 3186 & $0-500$ \\
\hline FII & $\%$ & 115 & 65 & 146 & $70-120$ \\
\hline $\mathrm{FV}$ & $\%$ & 102 & 55 & 172 & $70-120$ \\
\hline FVII & $\%$ & 88 & 50 & 173 & $70-120$ \\
\hline $\mathrm{FV} I I^{\mathrm{a}}$ & $\%$ & 261 & 94 & 343 & $70-150$ \\
\hline$v W F^{a}$ & $\%$ & 288 & 149 & 509 & $58-174$ \\
\hline FIX & $\%$ & 104 & 65 & 161 & $70-120$ \\
\hline FX & $\%$ & 84 & 41 & 122 & $70-120$ \\
\hline $\mathrm{FXI}$ & $\%$ & 85 & 42 & 138 & $70-120$ \\
\hline FXII & $\%$ & 100 & 54 & 125 & $70-120$ \\
\hline FXIII & $\%$ & 116 & 49 & 130 & $70-120$ \\
\hline Plasminogen & $\%$ & 88 & 44 & 119 & $75-150$ \\
\hline Leukocytes & $\times\left. 10^{9}\right|^{-1}$ & 8 & 3 & 13 & $4.0-10.0$ \\
\hline Erythrocytes $^{\mathrm{a}}$ & $\times\left. 10^{12}\right|^{-1}$ & 4.2 & 3.2 & 4.7 & $4.4-5.9$ \\
\hline Haemoglobin & $\left.g\right|^{-1}$ & 131 & 93 & 141 & $130-177$ \\
\hline Haematocrit $^{\mathrm{a}}$ & $1 \mathrm{I}^{-1}$ & 0.37 & 0.27 & 0.40 & $0.4-0.52$ \\
\hline Platelets & $\times 10^{9} \mathrm{I}^{-1}$ & 172 & 84 & 344 & $150-380$ \\
\hline
\end{tabular}

CRP C-reactive protein, $\mathrm{pH}$ potential hydrogen, $\mathrm{Fl}$ fibrinogen, blood coagulation factor I, immun immunological method, AT-III anti-thrombin III, FI-FXIII blood coagulation factors I-XIII, D-Dim d-dimers, vWF von Willebrand Factor. Highlighting: ${ }^{\text {a }}$ out of reference range

\section{Secondary endpoints}

Regarding thrombin generation in addition to reaching the primary endpoint (compare above), the ETP remained significantly elevated until $48 \mathrm{~h}$ after PCC administration (V8) and $\mathrm{C}_{\max }$ until $12 \mathrm{~h}$ after PCC administration (V6). Compare Table 1 and Fig. 2a/b.

None of the measured ROTEM ${ }^{\oplus}$ parameters significantly improved after administration of PCC. MCF INTEM significantly decreased by $3 \%(p=0.019)$ after PCC administration and remained significantly decreased until one hour after PCC administration (V3, data not shown). $\mathrm{CT}_{\text {EXTEM }}$ was significantly decreased only after V5 (compare Fig. 2c). Assessment of coagulation status following the administration of PCC showed a significant increase in Quick $^{\mathrm{PT}}(+28 \%)$ ten minutes after PCC administration (V2). The Quick value remained significantly increased, as compared to baseline (V1), until V9 or four days after administration of PCC (compare Fig. 2d). Moreover, PCC caused a significant decrease of $7 \%$ in aPTT. aPTT also remained significantly decreased until V2 (compare Table 2). 


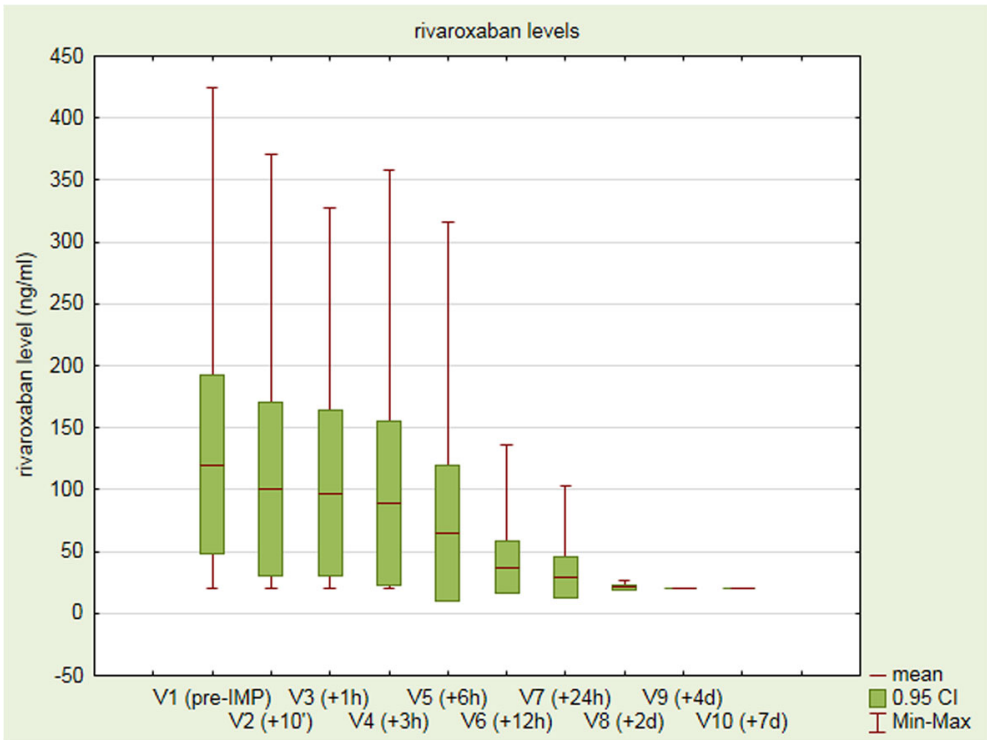

Fig. 1 Means of rivaroxaban levels from $\mathrm{V} 1$ to $\mathrm{V} 10$ for all included patients. Lowest rivaroxaban levels are indicated as $20 \mathrm{ng} \mathrm{ml}^{-1}$, because a lower level is not detectable

The single factor profiles showed a significant increase in activation of all coagulation factors contained in PCC (factors II, VII, IX and X) until V3 (FVII), V4 (FII) and until V7 (FIX and FX) as compared to baseline V1. All other coagulation factors showed no increase in activity following PCC administration (compare Additional file 1: Table S1).

Regarding correlation of rivaroxaban level with coagulation parameter, best correlation was found between $t_{\text {lag }}$ and rivaroxaban level (single measurements for every patient from V1 to V10 correlated with the respective rivaroxaban level) followed by $\mathrm{CT}_{\text {EXTEM }}, \mathrm{CT}_{\text {INTEM }}$, Quick value, and $\mathrm{C}_{\max }, \mathrm{MCF}_{\text {EXTEM}}$, and $\mathrm{t}_{\text {lag }}$. All other coagulation parameters showed no significant correlation with rivaroxaban levels (compare Table 3).

When comparing mean values of rivaroxaban and the mean values of all measured coagulation parameters at every time point $(V 1-V 10), t_{\text {lag }}, C_{\text {EXTEM }}, t_{\text {max }}$, MaxV$\mathrm{t}_{\text {FIBTEM }}, \quad$ MaxV-t $\mathrm{t}_{\text {EXTEM }}, \quad \mathrm{MCF}_{\text {FIBTEM }}, \mathrm{CT}_{\text {INTEM }}$ and $\mathrm{FI}_{\text {clauss }}$ showed a significant correlation with rivaroxaban levels. All other coagulation parameters showed no significant correlations with rivaroxaban levels (compare

Table 2 Coagulation parameters from V1 to V10

\begin{tabular}{|c|c|c|c|c|c|c|c|c|}
\hline Parameter & aPTT & Quick $^{P T}$ & ETP & $C_{\max }$ & $t_{\text {lag }}$ & $t_{\max }$ & $C_{\text {EXTEM }}$ & $\mathrm{CT}_{\text {INTEM }}$ \\
\hline Unit & $\mathrm{sec}$ & $\%$ & $\mathrm{mE}$ & $\mathrm{mE} \min ^{-1}$ & $\mathrm{sec}$ & $\mathrm{sec}$ & $\mathrm{sec}$ & $\mathrm{sec}$ \\
\hline Ref. range & $26-37$ & $70-130$ & $312-441$ & $111-156$ & $19.6-25.6$ & $50.8-72.0$ & $42-78$ & $134-218$ \\
\hline $\mathrm{V}_{1}$ & $30(6)$ & $72(16)$ & $316(75)$ & $80(31)^{*}$ & $52(70)^{*}$ & $99(67)^{*}$ & $94(31)^{*}$ & $178(37)$ \\
\hline V2 & $28(7)^{\ddagger}$ & $92(14)^{\ddagger}$ & $524(94)^{\ddagger^{*}}$ & $123(25)^{\ddagger}$ & $41(12)^{*}$ & $95(24)^{*}$ & $96(34)^{*}$ & $198(48)$ \\
\hline V3 & $29(6)^{\ddagger}$ & $89(11)^{\ddagger}$ & $502(104)^{\ddagger^{*}}$ & $114(28)^{\ddagger}$ & $39(9)^{*}$ & $99(29)^{*}$ & $91(25)^{*}$ & $180(41)$ \\
\hline V4 & $33(5)$ & $87(13)^{\ddagger}$ & $518(92)^{\ddagger^{*}}$ & $112(26)^{\ddagger}$ & $37(7)^{*}$ & $93(25)^{*}$ & $95(25)^{*}$ & $193(29)$ \\
\hline V5 & $32(6)$ & $88(15)^{\ddagger}$ & $544(102)^{\ddagger^{*}}$ & $127(22)^{\ddagger}$ & $33(6)^{*}$ & $91(25)^{*}$ & $83(22)^{*}$ & 170 (37) \\
\hline V6 & $32(11)$ & $83(18)^{\ddagger}$ & $528(118)^{\neq^{*}}$ & $131(31)^{\ddagger}$ & $34(9)^{*}$ & $83(29)^{*}$ & $80(17)^{*}$ & $176(27)$ \\
\hline V7 & $33(6)$ & $79(13)^{\ddagger}$ & $466(72)^{\ddagger^{*}}$ & $115(24)$ & $31(5)^{*}$ & $82(16)^{*}$ & $82(17)^{*}$ & $185(32)$ \\
\hline V8 & $31(6)$ & $88(15)^{\ddagger}$ & $411(98)^{\ddagger}$ & $111(27)$ & $27(5)^{*}$ & $69(16)$ & $62(15)$ & $156(37)$ \\
\hline V9 & $34(6)$ & $96(16)^{\ddagger}$ & $380(96)$ & $95(26)^{\neq^{*}}$ & $28(3)^{*}$ & $64(4)$ & $53(20)$ & $163(28)$ \\
\hline V10 & $29(7)$ & $88(22)$ & 371 (98) & $103(26)^{*}$ & $26(4)^{*}$ & $59(5)$ & $70(17)$ & $156(37)$ \\
\hline
\end{tabular}

aPTT activated partial thromboplastin time, Quick ${ }^{P T}$ Quick value, prothrombin time, $m E$ milli extinctions, ETP endogenous thrombin potential, $C_{\text {max }}$ thrombin generation, peak thrombin generation; $t_{\text {lag }}$ thrombin generation, lag time until initiation, $t_{\max }$ thrombin generation, time to peak thrombin activity, $C T$, ROTEM ${ }^{\bullet}$, clotting time, EXTEM extrinsic coagulation pathway; INTEM, intrinsic clotting time. Wilcoxon signed-rank test was used to evaluate differences between baseline and samples from the same patient at V2-V10 (indicated as ${ }^{\ddagger}$ ). Values are indicated as mean values (+/- standard deviation). Highlighting: ${ }^{*}$ out of reference range, ${ }^{*}$ significantly changed after PCC administration (as compared to V1) 

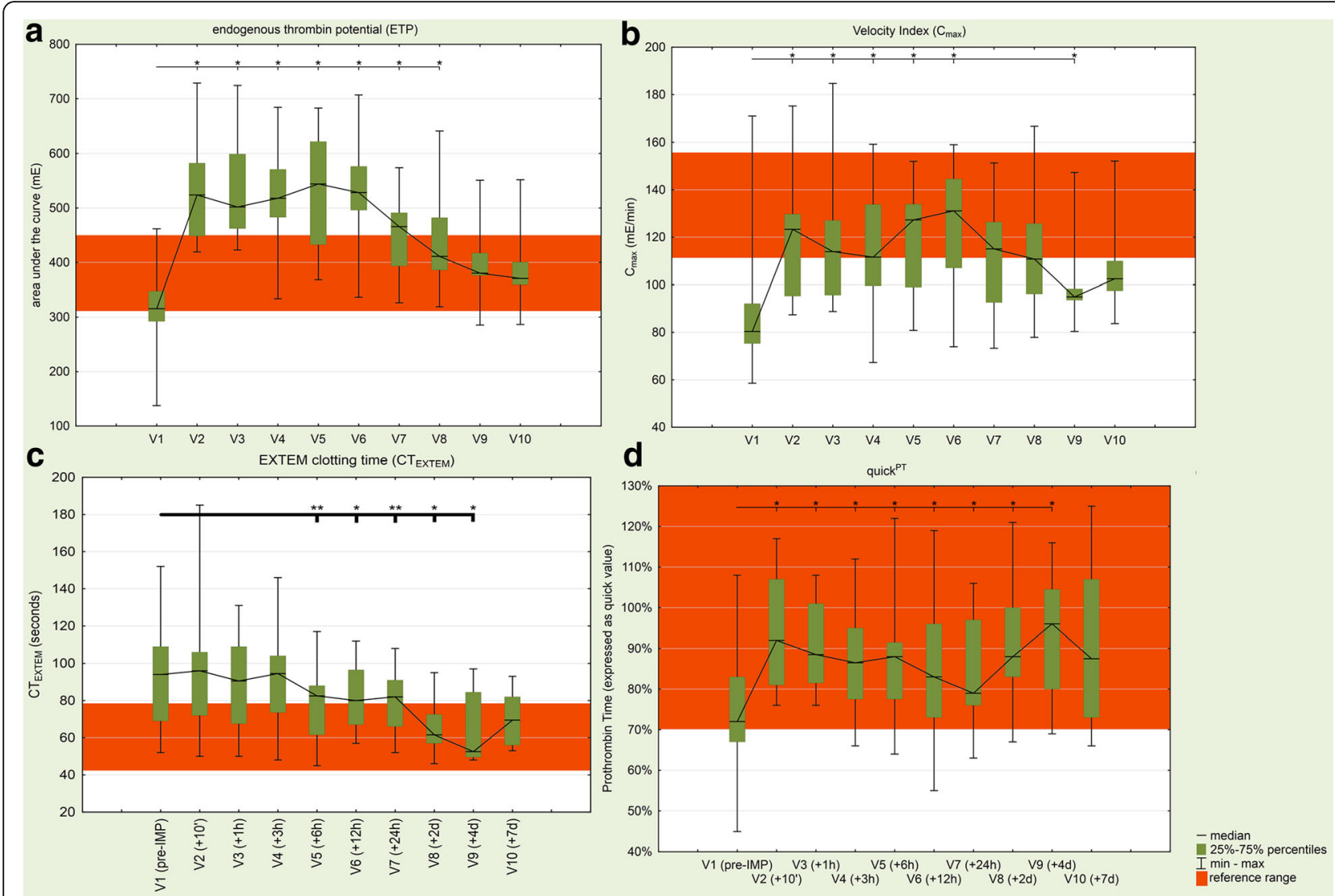

Fig. 2 Effect of four-factor PCC on coagulation parameters. ETP (a), $C_{\max }(\mathbf{b}), \mathrm{CT}_{\text {EXTEM }}$ (c) and Quick ${ }^{\text {PT }}$ (d) for all patients from V1 to V10. Normal (healthy persons without anticoagulation) values are indicated (red area). Significant differences from baseline were determined using the Wilcoxon signed-rank test $\left.{ }^{*} p<0.05,{ }^{* *} p<0.01\right)$. Abbreviations: ETP, endogenous thrombin potential; $C_{\max }$ thrombin generation, peak thrombin generation; $C T$, ROTEM$^{\circledast}$, clotting time; EXTEM, ROTEM ${ }^{\circledR}$, extrinsic coagulation pathway; Quick ${ }^{\text {PT }}$, Quick value (prothrombin time)

Table 3, Fig. 3). For values of all measured laboratory parameters, please refer to Additional file 1: Table S1.

\section{Discussion}

The present study enrolled patients with the need for acute reversal of the effects of rivaroxaban to investigate the effects of PCC on the coagulation status. The majority of included patients (77\%) suffered from lifethreatening bleeding events in the central nervous system $(\mathrm{ICH}$ or $\mathrm{SDH})$. Administration of PCC resulted in improved thrombin generation $\left(\mathrm{C}_{\max }, \mathrm{ETP}\right)$, aPTT and Quick $^{\mathrm{PT}}$, whereas ETP, aPTT and Quick ${ }^{\mathrm{PT}}$ were in normal range already before treatment. Therefore, $C_{\max }$ was the only parameter reduced at baseline (out of normal range) that improved by PCC administration. $\mathrm{T}_{\mathrm{lag}}, \mathrm{CT}_{\mathrm{EX}-}$ TEM and $t_{\max }$ correlated best with measured rivaroxaban levels and were out of normal ranges at baseline, but did not respond to PCC administration. Study design and the small number of included patients do not allow a profound safety evaluation to be conducted. However, ultrasound examination seven days after administration of four-factor PCC revealed no thrombotic events. The occurrence of thrombosis found in the present study was $14 \%$ during the observation period (30 days), which is comparable to the frequency found in the ANNEXA-4 study with an incidence of $18 \%$ [21].

To date, no studies regarding the efficacy of PCC in patients with life-threatening bleeding events, associated with DOACs, are available. Yoshimura and colleagues recently published data from an observational study evaluating PCC in ten patients with bleeding complications associated with DOAC treatment: PT also significantly improved after PCC administration, but in contrast to our study they found no significant changes in aPTT [22]. This might be attributed to the lower dose of PCC administered (median $1000 \mathrm{IU}$ vs. $2000 \mathrm{IU}$ in the present study). A recent retrospective study by Grandhi and colleagues investigated the effect of PCC on $\mathrm{ICH}$ in 16 patients under rivaroxaban treatment. They collected no data about coagulation status but found PCC to be effective in reducing haemorrhagic complications and hematoma expansion [12]. Erenberg and colleagues conducted a randomized, double-blind, placebo-controlled study with 12 healthy volunteers who 
Table 3 Correlations between rivaroxaban concentrations and blood coagulation parameters

\begin{tabular}{|c|c|c|c|c|c|}
\hline \multicolumn{3}{|c|}{ Individual values } & \multicolumn{3}{|l|}{ Mean values } \\
\hline parameter & $r$ & $\mathrm{p}$ & parameter & r & $\mathrm{p}$ \\
\hline$t_{\max }$ & 0.465 & $<0.001$ & $t_{\text {lag }}$ & 0.924 & $<0.001$ \\
\hline$C T_{\text {EXTEM }}$ & 0.355 & $<0.001$ & $\mathrm{CT}_{\text {EXTEM }}$ & 0.916 & $<0.001$ \\
\hline $\mathrm{CT}_{\text {INTEM }}$ & 0.296 & 0.002 & $t_{\max }$ & 0.902 & $<0.001$ \\
\hline Quick $^{\text {PT }}$ & -0.294 & 0.002 & MaxV-tFIBTEM & 0.887 & 0.001 \\
\hline$C_{\max }$ & -0.242 & 0.019 & MaxV-teXtem & 0.819 & 0.004 \\
\hline MCF EXTEM & 0.238 & 0.015 & $M_{\text {FIBTEM }}$ & 0.779 & 0.008 \\
\hline$t_{\text {lag }}$ & 0.222 & 0.027 & $C_{\text {INTEM }}$ & 0.742 & 0.014 \\
\hline MaxV-t tFIBTEM $_{\text {I }}$ & 0.221 & 0.128 & $\mathrm{FI}_{\text {clauss }}$ & 0.701 & 0.024 \\
\hline$M C F_{\text {INTEM }}$ & 0.203 & 0.037 & MaxV-tiNTEM & 0.598 & 0.068 \\
\hline CFT $_{\text {EXTEM }}$ & 0.194 & 0.047 & $\mathrm{Fl}_{\text {immun }}$ & 0.579 & 0.080 \\
\hline MaxV-teXteM & 0.172 & 0.242 & ATIII $\left.\right|_{X a}$ & 0.533 & 0.112 \\
\hline CFT INTEM $_{\text {INTE }}$ & 0.139 & 0.156 & MCF INTEM & 0.423 & 0.224 \\
\hline aPTT & 0.108 & 0.267 & Quick ${ }^{P T}$ & 0.340 & 0.337 \\
\hline MaxV-tinTEM & 0.070 & 0.635 & $\mathrm{CFT}_{\text {INTEM }}$ & 0.328 & 0.355 \\
\hline ETP & 0.025 & 0.809 & CFT $_{\text {EXTEM }}$ & 0.266 & 0.457 \\
\hline$M_{\text {CFIBTEM }}$ & 0.005 & 0.963 & aPTT & 0.185 & 0.609 \\
\hline
\end{tabular}

Individual values (left side, individual values for every single patient and for the respective test that was measured at every time point from V1 to V10) or mean values (right side, mean values of all patients and mean values of the measured rivaroxaban levels from V1 to V10) of all coagulation tests for all patients at every visit were correlated with the respective rivaroxaban concentrations at the same visit. The Pearson correlation was used to detect correlations between various blood coagulation tests and rivaroxaban concentrations. Tests were sorted according to the strength of correlation ( $r$ ). Abbreviations: $t_{\max }$ thrombin generation, time to peak, $C T, R O T E M^{\oplus}$ clotting time, EXTEM, ROTEM ${ }^{\circledR}$ extrinsic coagulation pathway, INTEM ROTEM ${ }^{\circledR}$, intrinsic clotting time, Quick ${ }^{P T}$ Quick value (prothrombin time), $C_{\max }$ thrombin generation, peak thrombin generation, MCF ROTEM ${ }^{\circledR}$, maximum clot firmness; $t_{\text {lag }}$ thrombin generation, lag time until initiation, $\operatorname{MaxV}^{-t} \mathrm{ROTEM}^{\circ}$, time from reaction start until the maximum of the first derivate of the curve is reached, FIBTEM ROTEM ${ }^{\ominus}$, fibrinogen-dependent coagulation, CFT ROTEM ${ }^{\circledR}$, clot formation time, aPTT activated partial thromboplastin time, ETP endogenous thrombin potential

received rivaroxaban for two and a half days $(2 \times 20 \mathrm{mg})$ and subsequently a PCC bolus $\left(50 \mathrm{U} \mathrm{kg}^{-1}\right)$ : effects of rivaroxaban and PCC were evaluated using the PT and ETP. Both parameters were affected by rivaroxaban and improved by PCC [10]. A similar study set up by Levi and colleagues with 35 healthy volunteers, who were randomized into a 4-factor PCC, a 3-factor PCC (50 U $\mathrm{kg}^{-1}$ for both) and a placebo (saline) group after four days of rivaroxaban $(2 \times 20 \mathrm{mg})$ treatment, also revealed that 4-factor PCC improved PT, aPTT and ETP [18]. In a recent study, we already demonstrated the effects of PCC on $\mathrm{CT}_{\text {EXTEM }}$ and TG ex vivo [16]: PCC was able to improve both parameters ex vivo, and rivaroxaban levels correlated with Quick ${ }^{\mathrm{PT}}, \mathrm{CTs}, \mathrm{C}_{\max }$, aPTT and $\mathrm{t}_{\mathrm{lag}}$.

The main difference between the present study and previous investigations is the different study set-up (in vivo). Consequently, rivaroxaban levels were more heterogeneous than in controlled ex vivo studies with patient blood or in vitro studies with healthy volunteers. For example, in the present study ETP was not reduced (as compared to normal values) at baseline V1, but the exact time of rivaroxaban intake was mostly not known. Consequently, rivaroxaban levels were lower than in comparable studies and ETP was not impaired.

The majority of patients were admitted with acute intracerebral haemorrhage. Early hematoma enlargement occurs more frequently in patients on anticoagulant treatment, and is associated with worsened long-term outcome [23]. Therefore, immediate reversal of any anticoagulant is recommended. Although we did not include a clinical or radiographic outcome parameter in the current study, we were able to show that PCC can be used to improve coagulation in these patients.

Interestingly, factor VIII and VWF levels were shown to be strongly increased $(>200 \%)$ at all investigated time points. We already observed this phenomenon in previous investigations with patients who were not bleeding under rivaroxaban [16]. A potential explanation is that blood coagulation factor VIII and VWF levels significantly increase during an acute phase response [24].

Limitations of the present study are the lack of randomization / a control group and the small number of patients, resulting in insufficient data on mortality or effective haemostasis. However, this is the first prospective clinical study investigating the effect of PCC on rivaroxaban-related, life-threatening bleeding in vivo. Previous investigations have focused on healthy volunteers receiving an oral Xa inhibitor followed by PCC. To obtain these data at precise time points in emergency patients is challenging and not possible in a large number of patients except in an industry-sponsored study with research staff available around the clock. Especially the measured laboratory parameters add valuable knowledge to this topic.

Nevertheless, great care has to be taken since patients concomitantly took other medication, which probably influenced the results. Moreover, the influence of rivaroxaban on PT, aPTT, coagulation factor levels and thromboelastometry assays differs widely depending on the reagents used and must be evaluated for each particular reagent. Consequently, effects of rivaroxaban on the various assays can also be expected to differ from reagent to reagent.

Consequently, additional studies focusing on these outcomes must be conducted. Even though a specific antidote for the reversal of rivaroxaban is in development, there is still little knowledge about the real efficacy of Andexanet Alpha: preliminary analysis of the ANNEXA-4 study showed 79\% efficacy. However, four hours after administration of the antidote rivaroxaban levels returned almost to pre-treatment values [21]. Moreover, it is still unclear whether smaller hospitals 

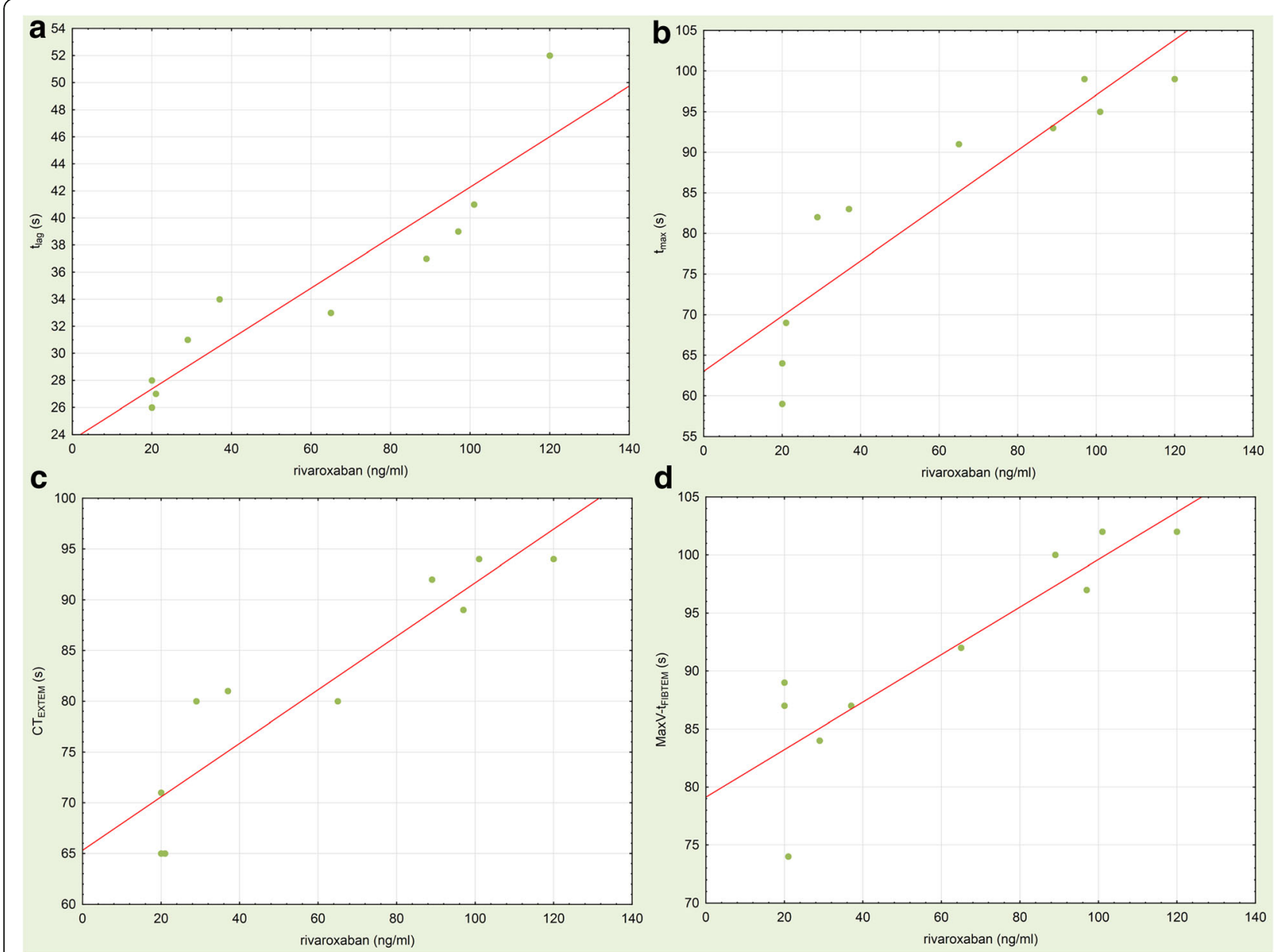

Fig. 3 Correlations between means of rivaroxaban plasma levels and various coagulation parameters from V1 to V10. Significantly ( $p<0.05$ for all) correlated parameters were (a) thrombin generation lag time ( $\mathrm{t}_{\text {lag, }}$ linear approximation for $\mathrm{t}_{\text {lag }}=23.63+0.19^{*} x, r=0.924, p<0.001$ ); (b) thrombin generation time to peak ( $t_{\text {max }}$ linear approximation for $\left.t_{\max }=63.02+0.34^{*} x, r=0.903, p<0.001\right)$; (c) EXTEM clotting time $\left(C T_{\text {EXTEM}}\right.$, linear approximation for $\left.C T=65.312+0.26^{*} x, r=0.926, p<0.001\right)$ and $(\mathbf{d})$ thromboelastometry FIBTEM time to maximum velocity (MaxV-t FIBTEM$_{\text {, linear }}$ approximation for MaxV-t FIBTEM $_{1}=79.132+0.205^{*} x, r=0.887, p<0.001$ ). Strength of correlation was interpreted by evaluating the correlation coefficient: $r$ 0.9-1.0, very strong correlation; r $0.7-0.89$, strong correlation; r 0.5-0.69, moderate correlation; r 0.3-0.49, weak correlation

will be able to store these, presumably very expensive, antidotes. Additionally, specific antidotes that directly target and eliminate the anticoagulant activity of the DOACs do not possess pro-coagulant properties, which might be necessary in patients suffering from severe haemorrhage. This indicates that coagulation factor concentrates should still be part of the management strategy in bleeding patients.

\section{Conclusions}

No controlled clinical studies in humans involving potential reversing agents in bleeding situations are available yet, which illustrates a difficulty in evaluating the potential reversing agents and proposing them for the management of life-threatening bleeding in rivaroxabananticoagulated patients. Our data indicate that fourfactor PCC might be a valuable alternative to specific antidotes for the reversal of rivaroxaban-induced and life-threatening bleeding events. Further studies with clinical endpoints will have to confirm these findings.

\section{Additional file}

Additional file 1: Table S1. Data are indicated as means (+/-standard deviation). Abbreviations: CRP, C-reactive protein; Fl, fibrinogen; immun, immunologic method; ATIII, anti-thrombin III; Flla, activated blood coagulation factor Ila; FXa, activated blood coagulation factor Xa; FIl, blood coagulation factor II; FV, blood coagulation factor $V$, etc.; VWF, Von Willebrand factor. (DOCX 23 kbr)

\section{Acknowledgments}

We sincerely thank the medical doctors and colleagues at the Medical University of Innsbruck, namely P. Innerhofer, M. Luger, B. Treichl, M. Joannidis, M. Ortler, B. Pfausler, A. Schiefecker, C. Reif, C. Irsara and P. Schech, who showed great commitment to study management and/or conduct. 


\section{Funding}

This work and the study drug were supported by an unrestricted grant provided by CSL Behring, Marburg, Germany. CSL Behring did not contribute in any way to the design of the study or to collection, analysis, and interpretation of data or the writing of the manuscript.

\section{Availability of data and materials}

All data generated or analysed during this study are included in this published article (and its supplementary information files). Moreover, the datasets used and/or analysed during the current study are available from the corresponding author on reasonable request.

\section{Authors' contributions}

BS, DF and MB conceived the study, designed the trial and wrote the applications to the ethics committee and to the public authorities. BS supervised the conduct of the trial and data collection. SG, RB and $\mathrm{RH}$ undertook recruitment of participating centres and patients. BS, SG, RB and $\mathrm{RH}$ are responsible for acquisition and management of the data, including quality control. BS and MB performed the laboratory experiments. BS and DF performed the statistical data analysis and interpretation. BS drafted the manuscript, and all authors contributed substantially to its critical revision. BS takes responsibility for the paper as a whole. All authors read and approved the final manuscript.

\section{Ethics approval and consent to participate}

This study was approved by the Human Subjects Review Board of the Medical University of Innsbruck, Austria (Ref.: UN2013-0048), as well as by the national competent authority (Bundesamt für Sicherheit im Gesundheitswesen, BASG, Vienna, Austria, Ref.: LCM-718199) and registered with EudraCT (Ref.: 2013-004484-31). Written informed consent was obtained from all study participants. Patients, who for medical reasons (unconscious, shock, sedated, etc.) were not able to give their consent to participate in this clinical trial, were enrolled based on the principle of deferred consent according to $\S 43 a$ (1), Austrian Medicinal Products Law ("Österreichisches Arzneimittelgesetz")

The study was performed in compliance with the Declaration of Helsinki guidelines regarding ethical principles for medical research involving human subjects and followed Good Clinical Practice as defined by the International Conference on Harmonization (ICH-GCP).

\section{Consent for publication}

Not applicable.

\section{Competing interests}

D. Fries has received study funding, honoraria for consultancy and board activity from Astra Zeneca, AOP orphan, Baxter, Baer, BBraun, Biotest, CSL Behring, Delta Select, Dae Behring, Edwards, Fresenius, Glaxo, Haemoscope, Hemogem, Lilly, LFB, Mitsubishi Pharma, NovoNordisk, Octapharm, Pfizer, Tem-Innovation.

R. Beer received speaker's honoraria from Boehringer Ingelheim RCV Gmbh \& Co KG. All other authors declare that they have no conflicts of interest.

\section{Publisher's Note}

Springer Nature remains neutral with regard to jurisdictional claims in published maps and institutional affiliations.

\section{Author details}

${ }^{1}$ Department of General and Surgical Intensive Care Medicine, Medical University of Innsbruck, Anichstr. 35, 6020 Innsbruck, Austria. ${ }^{2}$ Department of Neurosurgery, Medical University of Innsbruck, Anichstr. 35, Innsbruck 6020, Austria. ${ }^{3}$ Department of Anatomy, Histology and Embryology, Division of Clinical and Functional Anatomy, Medical University of Innsbruck, Anichstr. 35, Innsbruck 6020, Austria. ${ }^{4}$ Department of Neurology, Medical University of Innsbruck, Anichstr. 35, 6020 Innsbruck, Austria. ${ }^{5}$ Institute for Sports Medicine, Alpine Medicine and Health Tourism, UMIT - University for Health Sciences, Medical Informatics and Technology, Eduard Wallnöfer Zentrum 1, 6060 Hall in Tirol, Austria.
Received: 24 August 2017 Accepted: 21 December 2017

Published online: 10 January 2018

\section{References}

1. Haas S, Spannagl M, Schellong SM. Novel oral anticoagulants-key messages for the angiologist. Vasa. 2012;41:177-91.

2. Eikelboom JW, Weitz J. New anticoagulants. Circulation. 2010;121:1523-32.

3. Varughese CJ, Halperin JL. Prevention of stroke in patients with atrial fibrillation : anticoagulant and antiplatelet options. J Interv Card Electrophysiol. 2012;22:22.

4. McDonald CJ, Kalisch Ellett LM, Barratt JD, Caughey GE, Cross-Country Comparison A. Of rivaroxaban spontaneous adverse event reports and concomitant medicine use with the potential to increase the risk of harm. Drug Saf. 2014;1:1

5. Kaatz S, Kouides PA, Garcia DA, Spyropolous AC, Crowther M, Douketis JD, Chan AK, James A, Moll S, Ortel TL, et al. Guidance on the emergent reversal of oral thrombin and factor Xa inhibitors. Am J Hematol. 2012;87:4

6. Ferrandis R, Castillo J, de Andrés J, Gomar C, Gómez-Luque A, Hidalgo F, Llau JV, Sierra P, Torres LM. The perioperative management of new direct oral anticoagulants: a question without answers. Thromb Haemost. 2013;110:515-22.

7. Siegal DM, Curnutte JT, Connolly SJ, Lu G, Conley PB, Wiens BL, Mathur VS, Castillo J, Bronson MD, Leeds JM, et al. Andexanet Alfa for the Reversal of Factor Xa Inhibitor Activity. N Engl J Med.

8. Siegal DM, Garcia DA, Crowther MA. How I treat target-specific oral anticoagulant-associated bleeding. Blood. 2014;123:1152-8.

9. Levi M. Management of bleeding in patients treated with direct oral anticoagulants. Crit Care. 2016;20:249.

10. Eerenberg ES, Kamphuisen PW, Sijpkens MK, Meijers JC, Buller HR, Levi M. Reversal of rivaroxaban and dabigatran by prothrombin complex concentrate: a randomized, placebo-controlled, crossover study in healthy subjects. Circulation. 2011;124:1573-9.

11. Levi M, Moore KT, Castillejos CF, Kubitza D, Berkowitz SD, Goldhaber SZ Comparison of three-factor and four-factor prothrombin complex concentrates regarding reversal of the anticoagulant effects of rivaroxaban in healthy volunteers. J Thromb Haemost. 2014;12.

12. Grandhi R, Newman WC, Zhang X, Harrison G, Moran C, Okonkwo DO, Ducruet AF. Administration of 4-factor prothrombin complex concentrate as an antidote for intracranial bleeding in patients taking direct factor $\mathrm{Xa}$ inhibitors. World Neurosurg. 2015;84:1956-61.

13. Beynon C, Sakowitz OW, Storzinger D, Orakcioglu B, Radbruch A, Potzy A, Unterberg AW. Intracranial haemorrhage in patients treated with direct oral anticoagulants. Thromb Res. 2015;136:560-5. https://doi.org/10.1016/j. thromres.2015.07.001. Epub 2015 July 2016

14. Kauffmann S, Chabanne R, Coste A, Longeras F, Sinegre T, Schmidt J, Samama CM, Constantin JM, Lebreton A. Favorable outcome of rivaroxaban-associated intracerebral hemorrhage reversed by 4-factor prothrombin complex concentrate: impact on thrombin generation. A A Case Rep. 2015:4:151-4.

15. Thomas L. Labor und diagnose. 8th ed. Frankfurt: TH-Books Verlagsgesellschaft; 2012

16. Schenk B, Wurtinger $P$, Streif W, Sturm W, Fries D, Bachler M. Ex vivo reversal of effects of rivaroxaban evaluated using thromboelastometry and thrombin generation assay. Br J Anaesth. 2016;117:583-91.

17. Eerenberg ES, Kamphuisen PW, Sijpkens MK, Meijers JC, Buller HR, Levi M. Reversal of rivaroxaban and dabigatran by prothrombin complex concentrate: a randomized, placebo-controlled, crossover study in healthy subjects. Circulation. 2011;124

18. Levi M, Moore KT, Castillejos CF, Kubitza D, Berkowitz SD, Goldhaber SZ, Raghoebar M, Patel MR, Weitz J, Levy JH. Comparison of three-factor and four-factor prothrombin complex concentrates regarding reversal of the anticoagulant effects of rivaroxaban in healthy volunteers. J Thromb Haemost. 2014;12:1428-36.

19. Dinkelaar J, Molenaar PJ, Ninivaggi M, de Laat B, Brinkman HJ, Leyte A. In Vitro assessment, using thrombin generation, of the applicability of prothrombin complex concentrate as an antidote for rivaroxaban. J Thromb Haemost. 2013;11:1111-8.

20. Gerotziafas GT, Elalamy I, Depasse F, Perzborn E, Samama MM. In Vitro inhibition of thrombin generation, after tissue factor pathway activation, by the oral, direct factor Xa inhibitor rivaroxaban. J Thromb Haemost. 2007;5(4): 886-8. Epub 2007 Feb 7. 
21. Connolly SJ, Milling TJJ, Eikelboom JW, Gibson CM, Curnutte JT, Gold A, Bronson MD, Lu G, Conley PB, Verhamme P, et al. Andexanet alfa for acute major bleeding associated with factor Xa inhibitors. N Engl I Med. 2016;375:1131-41.

22. Yoshimura S, Sato S, Todo K, Okada Y, Furui E, Matsuki T, Yamagami H, Koga M, Takahashi JC, Nagatsuka K, et al. Prothrombin complex concentrate administration for bleeding associated with non-vitamin $\mathrm{K}$ antagonist oral anticoagulants: the SAMURAI-NVAF study. J Neurol Sci. 2017;375:150-7.

23. Steiner T, Al-Shahi Salman R, Beer R, Christensen H, Cordonnier C, Csiba L, Forsting M, Harnof S, Klijn CJ, Krieger D, et al. European stroke organisation (ESO) guidelines for the management of spontaneous intracerebral hemorrhage. Int J Stroke. 2014;9:840-55.

24. Jenkins PV, Rawley O, Smith OP, O'Donnell JS. Elevated factor VIII levels and risk of venous thrombosis. Br J Haematol. 2012;157:653-63.

Submit your next manuscript to BioMed Central and we will help you at every step:

- We accept pre-submission inquiries

- Our selector tool helps you to find the most relevant journal

- We provide round the clock customer support

- Convenient online submission

- Thorough peer review

- Inclusion in PubMed and all major indexing services

- Maximum visibility for your research

Submit your manuscript at www.biomedcentral.com/submit
Biomed Central 